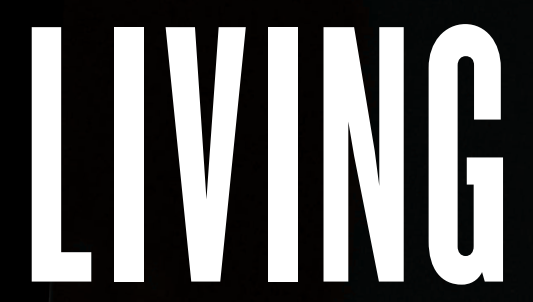

\title{
OW THE
}

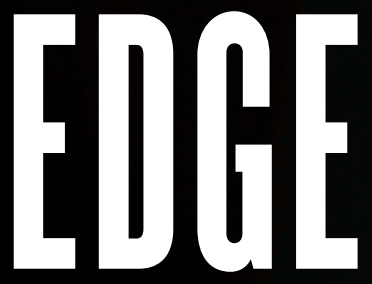

\section{By pushing materials to new limits, Gil Lonzarich sparked a revolution in the study of quantum states of matter.}

\section{BY ELIZABETH GIBNEY}

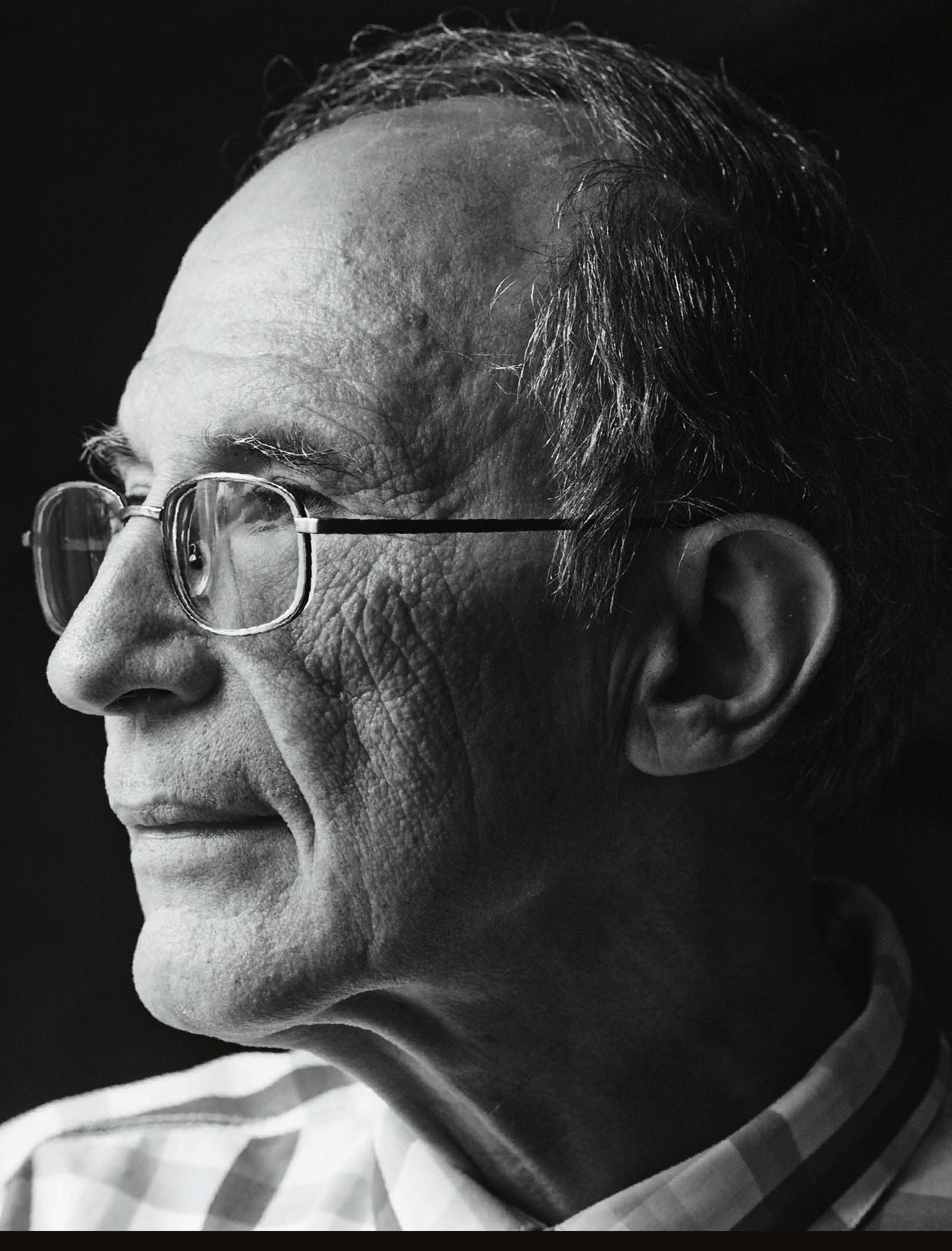

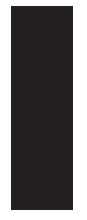

n 1989, surgery for detached retinas left Gilbert Lonzarich blind for a month. Rather than feel shaken or depressed, the condensedmatter physicist at the University of Cambridge, UK, seized the opportunity, inviting his graduate students to his house to share with them how exciting it was to adapt to life without sight. Lonzarich's embrace of the experience perfectly captures his approach to life, says Andrew Mackenzie, then one of those students and now a director at the Max Planck Institute for Chemical Physics of Solids in Dresden, Germany. "Gil is one of the most positive people I've ever met. He finds interest in everything," he says.

For more than 40 years, that optimism and curiosity has led Lonzarich to probe materials in ways never thought possible. In pioneering experiments in the 1990s, his team showed that pushing magnetic compounds to extreme pressures and close to absolute zero can make some of them conduct electricity without resistance ${ }^{1}$. This flew in the face of convention, which declared that magnetism and superconductivity could never mix. "It was as if nowadays you were talking about finding aliens or something," says Malte Grosche, a colleague at Cambridge.
That work showed physicists a new way to hunt for superconductors, which lie at the heart of technologies such as magnetic resonance imagers and particle accelerators. In recent years, it has offered a potential explanation for why some materials remain superconductors at temperatures much higher than absolute zero, which could pave the way to developing efficient, cheap devices that superconduct at room temperature.

But the experiments have had an impact well beyond superconductivity. Lonzarich's method of subjecting materials to extreme conditions has become a general recipe for discovering new states of matter. Around the world, physicists now use this approach to probe a range of materials in which the collective interactions of electrons can give rise to unusual behaviour. Some of these phenomena could potentially revolutionize computing.

Lonzarich's research may be legendary in his community, but the physicist's humility and generosity are what endear him to his colleagues. He is famously unconscious of time; a casual conversation with Lonzarich can easily lead to an hours-long random walk through the byways 
of physics, philosophy, politics and history. That might mean a missed lunch, says Michael Sutherland, a Cambridge colleague - "but it's the most productive few hours you'll have all week". Phone calls with peers frequently last into the small hours of the morning, and on the rare occasions when Lonzarich goes to a conference, he invariably attracts a mass of fellow attendees. "People who meet him even once or twice develop a sense of attachment and awe," says Louis Taillefer, another former student, who is now a physicist at the University of Sherbrooke in Canada.

At 72, Lonzarich now has a part-time role in the Cambridge quantummatter group, but he is still making new discoveries by pushing materials to ever-greater extremes. He sees this little-explored realm as just as fundamental to unravelling the laws of physics as the high-energy experiments at particle colliders, and expects that there is plenty more to discover. "Gil has never believed that we're now just filling in the details," says Piers Coleman, a theoretical physicist at Rutgers University in Piscataway, New Jersey. "He really views the exploration of quantum matter as a true frontier."

\section{COLLECTIVE EFFORTS}

Walking around a timeworn study at Trinity College, Cambridge, Lonzarich is eager to point out a portrait of the economist John Kenneth Galbraith, one of his heroes, and he talks enthusiastically about the impressive work of his colleagues. But when conversation turns to his own achievements, Lonzarich becomes reticent. It is human nature is to celebrate heroes, he says, but science is a collective activity, and singling out individuals for praise stifles a team.

Although colleagues are quick to highlight Lonzarich's influence, he never would - a practice that could be traced back to his upbringing, by Italian parents, on the Istria peninsula. His father told him to "always cut the larger slice of the pie for the other person", he recalls. At school, he learnt about the Roman Republic and was intrigued by the importance placed on reason, compromise and collaborative governance.

His family moved to the United States when he was nine. By the 1960s, Lonzarich had grown into a studious young man. His interest in physics began at the University of California, Berkeley, where he earned a liberal arts degree. It was there that he met Gerie Simmons (now Lonzarich). The pair had admired each other from afar before she engineered their meeting by pretending to need a physics tutor; they married in 1967.

"On the rare occasions I've seen pictures of him from those days, he had long hair. He was a physics hippy," says Coleman. But although Lonzarich felt strongly that people should challenge the government, including the United States' nascent war in Vietnam, he became disillusioned with the counter-culture's free use of drugs and rejection of family. "I wanted to be able to do something tangible, to make good use of life. I didn't think we were doing that," he says.

After a they spent a spell at the University of Minnesota in Minneapolis, the darker side of the movement eventually drove Gerie and Gil away from the United States altogether, to the University of British Columbia in Vancouver, Canada. There, Lonzarich became fascinated with magnetism, while working on his $\mathrm{PhD}$ in a new laboratory led by condensed-matter physicist Andrew Gold. When he left in 1976 for a stint at Cambridge, he found his new Rome. The collegiate structure had no real hierarchy and boasted two giants of condensed-matter physics - Brian Pippard and David Shoenberg. What was intended to be a one-year European adventure ended up lasting more than 40 years.

Lonzarich arrived at Cambridge wanting to study magnets - materials in which the spins of electrons all spontaneously align. His approach raised a few eyebrows at first: he developed his own mathematical notation and would spend weeks preparing his experiments while seeming to do nothing. But his methods soon began to bear fruit.

In magnetic materials, spins maintain their orderly arrangement only up to a point; above a certain temperature, electrons have so much energy that they can easily overcome the forces that cause their spins to align. Lonzarich reckoned that the best way to understand magnetic materials was to push them to that point, where they would be poised on the knife-edge between order and disorder. In particular, he was interested in exploring what might happen if the magnetic transition were shifted so that quantum effects could potentially alter the material's state. At higher pressures, the transition occurs at lower temperatures. And with enough pressure, a material can be 'tuned' so that its magnetic transition point occurs close to absolute zero. Here, thermal vibrations don't provide enough energy for the material to lose its magnetic order. Instead, quantum fluctuations - transient changes in electron properties, such as velocity and position, caused by the inherent uncertainty of the quantum world - dominate and can cause the material to switch states. In this regime, a region around a spot at absolute zero called the quantum critical point, magnetic materials become unstable and teeter on the brink of magnetism: they lack order but itch to align.

With larger physical forces suppressed around the quantum critical point, ordinarily weak interactions between electrons could have huge effects. And they might, Lonzarich reasoned, give rise to new states of matter through their collective interaction. "It's like in a forest; the little plants won't grow until the big tree is cut down," he says.

In particular, Lonzarich predicted that antiferromagnets - magnetic materials in which neighbouring spins align in opposite directions below a certain temperature - would become superconducting near the quantum critical point. On the verge of magnetism, he reasoned, the electrons would be so eager to align that they might spontaneously form pairs with opposite spins. Such antiparallel pairs would stick together, and their attraction to each other would stabilize their journey through the material's atomic lattice (see 'Hidden powers').

Since the mid-1980s, various theorists had suggested that such magnetically mediated superconductivity could arise, but Lonzarich's team was the first to provide solid experimental proof. When the group pushed a sample of the antiferromagnet cerium indium-3 close to the quantum critical point by cooling it at high pressure, the researchers saw it flip into a superconducting phase - something never before seen in a magnetic material ${ }^{1}$.

The work, which was performed in 1994, demonstrated a new category of superconductor. It also provided a road map by which to search for other superconducting materials. Today, physicists routinely push the phase transition in magnetic materials down to absolute zero to see whether this behaviour emerges.

\section{NEW TERRAIN}

The quantum critical point, and the strong quantum interactions that can take place around it, can give rise to other exotic states, not just superconductivity. "It's like a breeding ground for discovering new states of matter," says Cambridge physicist Stephen Rowley. Physicists around the world now manipulate a range of different factors - pressure, magnetic fields and chemical composition - to push phase transitions towards lower temperatures and so approach a quantum critical point.

In the late 1990s, this method led Lonzarich and then-student Christian Pfleiderer to discover strange behaviour in the material manganese silicide ${ }^{2}$. Experiments done in the past few years have hinted that this may be connected to swirling two-dimensional magnetic vortices, known as skyrmions, that were later described by Pfleiderer and his colleagues ${ }^{3}$ and are now being touted as a superefficient way to store information. By probing around a quantum 


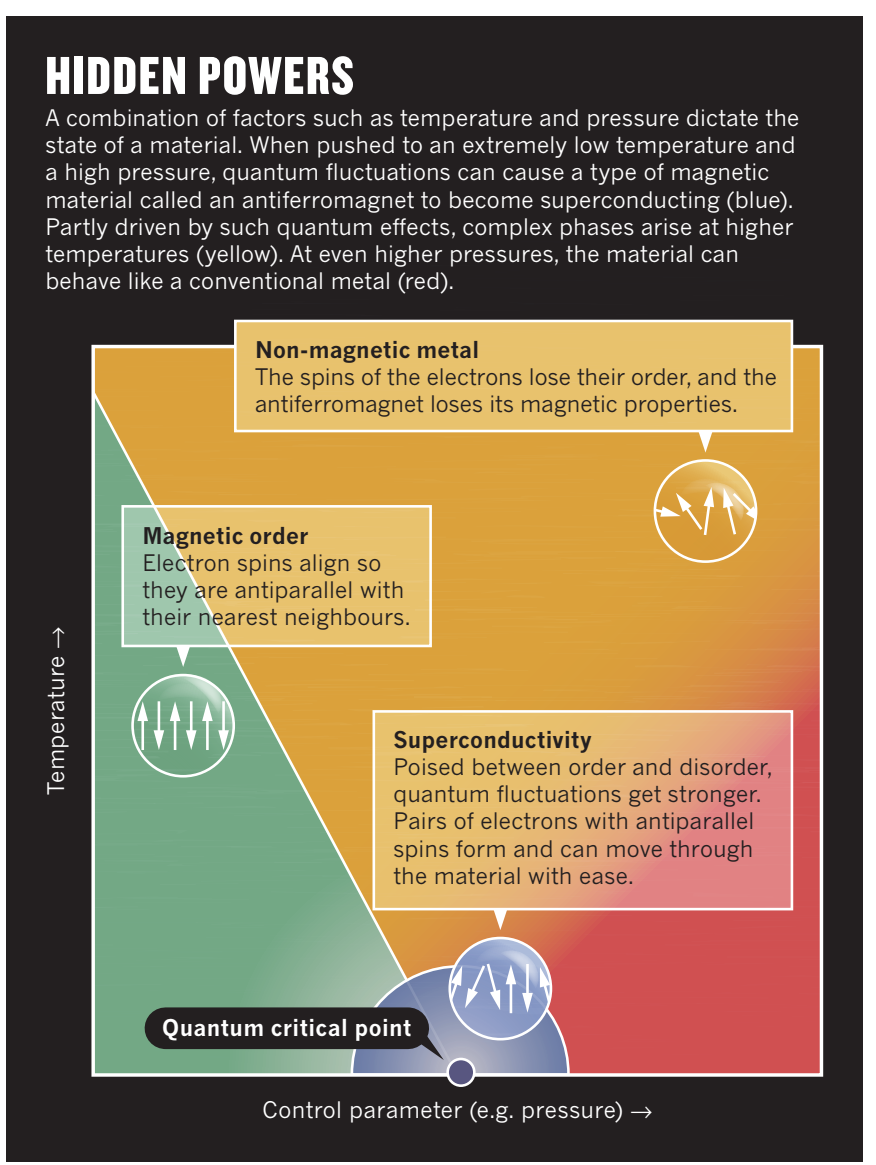

critical point of strontium ruthenate oxide, in 2007 Mackenzie and his team confirmed the existence of a new phase of matter, in which electrons flow but still show an orderly spatial structure ${ }^{4}$.

Fellow physicists say Lonzarich is unique in that he is not only a good theorist but also an exceptional experimenter. "You have to look back to Enrico Fermi to someone able to think so deeply about theory and do really good experiments," says David Pines, a physicist and distinguished research professor at the University of California, Davis. Lonzarich grows his own samples to extreme levels of purity and pioneered a technique, known as quantum oscillation, that allows physicists to determine the electronic structure of complex, interacting systems ${ }^{5}$. Patricia Alireza, who runs the high-pressure laboratory at Cambridge's Cavendish Laboratory, says that Lonzarich will often encourage her to create devices that squeeze samples well beyond what was thought possible. "Gil will smile and say, 'I think we could probably do a factor of 100 better than that", she says. "And you know what? We always do."

Many of Lonzarich's students have continued in physics and flourished. Suchitra Sebastian, for example, led work with Lonzarich a few years ago on samarium hexaboride, an insulator that exhibits metal-like behaviour when exposed to strong magnetic fields ${ }^{6}$. She says that without his advice she would probably have left the field. "He is not just teaching you 'this is how you do physics' but 'this is how to survive in the world of physics," she says. Lonzarich is modest about how much he contributed to the success of those he has mentored, saying that they taught him at least as much as the other way around.

One thing he always has for people is time, says Rowley. It helps that he is adept at escaping unnecessary bureaucracy, adds Pines. "He has many different offices so he can always hide at one." But Lonzarich's freedom to think is largely enabled by his wife, Gerie. She ensures that grant applications are handed in on time and that flights are caught. Gil says his wife is like the Sun: "So big and important that sometimes you forget it's the reason everything is there."

\section{LINGERING MYSTERY}

In the past few years, Lonzarich's ideas about the intimate link between superconductivity and magnetism have gained new relevance. Physicists explain conventional superconductivity using BCS theory ${ }^{7}$, named after the initials of the surnames of the three people who published it in 1957. The theory states that an electron speeding through some materials creates a positively charged distortion in the atomic lattice behind it. This pulls in a second electron, which follows the first like a cyclist riding in a competitor's slipstream. If enough of these relatively stable 'Cooper pairs' form, they create an ordered state in which the two electrons keep one another on course and flow without resistance.

But this explanation cannot account for sandwiches of copperbased insulators known as cuprates and for iron-based semiconductors. These two classes of superconductor can carry currents without resistance at temperatures up to 133 kelvin. If such transitions can be boosted to room temperature, around 300 kelvin, those superconductors could allow for cheaper energy, medical imaging and transportation. But debate about how they work has raged for 30 years.

From the start, one camp thought that magnetic interaction which can be more resilient to temperature than are interactions caused by distortions in the lattice - might somehow bind electrons together to create superconductivity in cuprates. Lonzarich theorized that this magnetic glue might stem from the same quantum fluctuations that ramp up around antiferromagnet quantum critical points. This idea is now hotly debated, and gained some supporting evidence last year in experiments conducted by Taillefer's team, with collaborators at the National Laboratory for Intense Magnetic Fields in Toulouse, France. The group found that stripping a cuprate of its superconductivity with a powerful magnetic field and adding increasing levels of impurities revealed a sharp phase transition an otherwise hidden quantum critical point ${ }^{8}$. Although the precise nature of that point is still not clear, it seems likely that antiferromagnetic correlations are at play, says Taillefer. "Which would mean Gil had a hell of an intuition," he says.

Lonzarich is now looking beyond conventional high-temperature superconductors. With Rowley and other colleagues, he is examining the nature of ferroelectrics, a little-studied class of ionic materials that generate their own electric field. At low temperature, ferroelectrics can become superconductors in a manner that parallels how superconductivity emerges in magnetic materials. Lonzarich has a hunch that in ferroelectric materials that also exhibit magnetism, electron pairs bind so strongly that the state could survive to room temperature.

The Universe is richer than most scientists give it credit for, Lonzarich says. Each newly discovered state of matter emerges only when conditions are right and a material is sufficiently pure. Lonzarich speculates that probing the boundaries around those states could reveal more phases, and studying the boundaries of those could reveal yet more, with discoveries unfolding in a fractal manner. "What if each quantum critical point is just the beginning of another generation? There's some indication we're heading in that direction," he says.

The idea is highly speculative, but Taillefer says people would be wise to listen. The notion that a now-familiar principle could hide deep, complex behaviour "is typical Gil", he says. "I would definitely put my money on him."

Elizabeth Gibney is a senior reporter for Nature in London.

\footnotetext{
1. Mathur, N. D. et al. Nature 394, 39-43 (1998).

2. Pfleiderer, C., McMullan, G. J., Julian, S. R. \& Lonzarich, G. G. Phys. Rev. B 55, 8330 (1997)

3. Mühlbauer, S. et al. Science 323, 915-919 (2009).

4. Borzi, R. A. et al. Science 315, 214-217 (2007).

5. Taillefer, L. \& Lonzarich, G. G. Phys. Rev. Lett. 60, 1570-1573 (1988),

6. Tan, B. S. et al. Science 349, 287-290 (2015).

7. Bardeen, J., Cooper, L. N. \& Schrieffer, J. R. Phys. Rev. 108, 1175-1204 (1957).

8. Badoux, S. et al. Nature 531, 210-214 (2016).
} 